\title{
Effects of black yeast-derived $\beta$-1,3-1,6-glucan on serum cytokine and microRNA expression in transplanted sarcoma in mice
}

\author{
WEI $\mathrm{LI}^{1}$, YARU ZHANG ${ }^{2}$ and FENGSONG CONG ${ }^{2}$ \\ ${ }^{1}$ Key Laboratory of Systems Biomedicine, Ministry of Education, Shanghai Center for Systems Biomedicine \\ and ${ }^{2}$ School of Life Sciences and Biotechnology, Shanghai Jiaotong University, Shanghai 200240, P.R. China
}

Received August 16, 2012; Accepted October 1, 2012

DOI: $10.3892 /$ br.2012.30

\begin{abstract}
. $\beta-1,3-1,6$-glucans are the most abundant glucose polymers in the cell walls of fungi. Previous studies have shown that $\beta-1,3-1,6$-glucans derived from fungi possess immunomodulating activitivies. Antitumor effects of these compounds have also been reported in animal models. Current studies mainly focus on the direct effects of $\beta-1,3-1,6$-glucans on immune systems, but no data are available to address the underlying molecular events in tumor cells. $\beta-1,3-1,6$-glucan purified from black yeast at $5 \mathrm{mg} / 100 \mathrm{~g}$ body weight (study group) or saline (control group) was intragastrically administered on a daily basis to subcutaneously-injected mice with mouse S180 sarcoma cells. Tumor sizes, tumor weights, serum concentrations of cytokines and levels of microRNAs (miRNAs) in transplanted tumors were compared between the treated and control groups. The volumes and weights of transplanted tumors were significantly lower in the treatment groups compared to the control groups by $\sim 150 \%$ and $70 \%$, respectively. The treated mice demonstrated significantly higher levels of cytokines, including IL-2, IL-4, IL-6, IL-8, IL-10 and IL-12, compared to the control mice. Notably, the expression of several miRNAs in transplanted tumor tissues also markedly changed. These data suggest that black yeast-derived $\beta-1,3-1,6$-glucan, not only stimulates cytokine release from immune cells, but also changes the expression profiles of miRNAs in transplanted tumors.
\end{abstract}

\section{Introduction}

$\beta$-glucans are some of the most abundant polysaccharides found in the cell walls of bacteria and fungi $(1,2)$. They are present in water-soluble or -insoluble forms, while certain $\beta$-glucans can be excreted into the medium (3). $\beta$-glucans

Correspondence to: Professor Fengsong Cong, School of Life Sciences and Biotechnology, Shanghai Jiaotong University, 800 Dongchuan Road, Minhang, Shanghai 200240, P.R. China

E-mail: fscong@sjtu.edu.cn

Key words: $\beta$-1,3-1,6-glucan, S180 sarcoma cell, transplanted tumor, cytokine, microRNA have been proven to act as immunological modulators (4). Their water-soluble form has been shown to have a more pronounced effect on immunity (5). They have the potential to directly bind to specific receptors on immune cells and trigger innate and/or adaptive immunity activation. Currently, a number of $\beta$-glucan receptors have been identified. Dectin-1, located on macrophages, mediates the $\beta$-glucan activation of phagogenesis and the production of cytokines (6,7). Activated complement receptors on natural killer cells, neutrophils and lymphocytes have also been suggested to be associated with cytotoxicity (8). Certain toll-like receptors (TLRs) on macrophage cells were also demonstrated to specifically bind with $\beta$-glucans and increase tumor necrosis factor (TNF)- $\alpha$ and interleukin (IL)-12 production via nuclear factor $-\kappa \mathrm{B}(\mathrm{NF}-\kappa \mathrm{B})$ signaling (9). Two additional receptors, scavenger $(10,11)$ and lactosylceramide (12), bind with $\beta$-glucans and mediate signal pathways leading to immunological activation.

The antitumor activity of $\beta$-glucans was first demonstrated 50 years ago. A number of subsequent experiments conducted in animals have confirmed the inhibitory effects of certain $\beta$-glucans on tumor growth or metastasis (13). Several clinical trials have also shown the potential benefits of $\beta$-glucans in the treatment of cancer patients (14). Fungal $\beta$-glucans have also been proven to have synergistic effects with monoclonal antibodies, when used in the treatment of certain types of cancer (15). Although one of the most well-known anticancer mechanisms is stimulation of innate immunity, direct action on tumor cells has also been suggested (16). Therefore, fungal $\beta$-glucans may exert multiple effects on cancer cells (4). Certain $\beta$-glucans have been clinically used as a complementary therapy for cancer in Japan and China, due to their ability to enhance host defense responses against various tumors.

Fungal $\beta$-glucans are present as $\beta$ - $1 \rightarrow 3$-linked glucose polymers with $\beta-1 \rightarrow 6$-linked side chains of varying lengths and distributions (2). Secreted yeast $\beta$-glucans are important sources of $\beta$-glucans for potential medical use, due to the fact that they can be recovered and purified more easily (3). Black yeast $\beta$-1,3-1,6-glucan belongs to the category of soluble glucans (17). Previous studies have shown a noteworthy stimulatory effect of this compound on the production of IL-8 by peripheral blood mononuclear cells (18). However, a limited number of studies has been conducted with a view to address the function of this compound on tumor growth (17). In this study, we demonstrated that gastric administration 
of purified black yeast $\beta$-1,3-1,6-glucan significantly inhibited transplanted sarcoma growth in mice. In addition to its proven role in immunity stimulation, the microRNA (miRNA) expression profiles in transplanted tumors also changed, due to $\beta-1,3-1,6$-glucan administration.

\section{Materials and methods}

Materials. $\beta$-1,3-1,6-glucan purified from black yeast was kindly provided by Gokei Trading Co., Ltd. (Tokyo, Japan). RPMI-1640 medium, fetal bovine serum (FBS), miRVana RNA isolation and mirVana ${ }^{\mathrm{TM}}$ miRNA isolation kits were purchased from Life Technologies (Carlsbad, CA, USA). Cytokine enzyme-linked immunosorbent assay (ELISA) kits were purchased from R\&D (Minneapolis, MN, USA), while isoflurane was obtained from Abbott Laboratories (North Chicago, IL, USA).

Cell line and animals. The mouse sarcoma S180 cell line was purchased from the Cell Bank of the Chinese Academy of Sciences (Shanghai, China). Twenty male BALB/c mice (6-week-old; $n=20$ ) were purchased from Shanghai Xipuerbikai Experimental Animal Co. Ltd. (Shanghai, China). The mice were kept in cages ( $\mathrm{n}=5$ per group), at $22 \pm 1^{\circ} \mathrm{C}$ and $55 \pm 5 \%$ relative humidity, with an automatic 12-h light/dark cycle. The experimental procedures were approved by the Animal Ethics Committee of the Shanghai Jiaotong University (Shanghai, China).

Animal experiment. Mice were randomized into the control and study groups ( $\mathrm{n}=10 /$ group). Mouse sarcoma $\mathrm{S} 180$ cells were cultured in RPMI-1640 medium containing 10\% FBS. Cells growing in log phase were trypsinized and resuspended in sterile saline and immediately inoculated into mice by subcutaneous injection $\left(2 \times 10^{6}\right.$ cells in $0.2 \mathrm{ml}$ saline/mouse). Immediately after the inoculation, mice in the control and study groups were submitted to daily intragastric administration of $\beta-1,3-1,6$-glucan ( $5 \mathrm{mg} / 100 \mathrm{~g}$ body weight) or saline, respectively. Nine days after the implantation, the engrafted tumor sizes were measured daily in vivo, using external caliber and were calculated as previously reported $(19,20)$. Twenty-one days after treatment, the mice were anaesthetized with $2 \%$ isoflurane and blood was drawn by cardiac puncture. Serum was separated from the clot within $20 \mathrm{~min}$ and stored at $-20^{\circ} \mathrm{C}$ until use. The mice were then sacrificed and engrafted tumors were dissected, weighed and frozen in liquid nitrogen. Tumor sizes and weights were calculated using the formula: $\mathrm{A} \%=[(\mathrm{X}-\mathrm{Y}) / \mathrm{X}] \mathrm{x} 100$, where $\mathrm{A}$ is the inhibition rate, $\mathrm{X}$ is the average tumor weight of control group and $\mathrm{Y}$ is the average tumor weight of the study group.

Measurement of cytokine content. The serum content of 10 cytokines including IL-2, IL-4, IL-6, IL-8, IL-10, IL-12, interferon (IFN) $-\gamma$, TNF- $\alpha$, TNF- $\beta$ and granulocyte-colony stimulating factor (G-CSF) were determined using double antibody sandwich ELISA tests, following the manufacturer's instructions. Two-way ANOVA was used to determine the significance of statistical differences in cytokine concentrations in the control and study groups. $\mathrm{P}<0.05$ was considered to indicate a statistically significant difference.
Table I. Correlation between body and tumor weight.

\begin{tabular}{lcc}
\hline & \multicolumn{2}{c}{ Groups } \\
\cline { 2 - 3 } Variables & Control $(\mathrm{n}=10)$ & Study $(\mathrm{n}=10)$ \\
\hline Body weight $(\mathrm{g})$ & $25.31 \pm 2.41$ & $23.60 \pm 1.46$ \\
Tumor weight $(\mathrm{g})$ & $3.081 \pm 0.998$ & $0.898 \pm 0.396^{\mathrm{b}}$ \\
Actual body weight $(\mathrm{g})$ & $22.23 \pm 2.678$ & $22.70 \pm 1.248$ \\
Tumor weight/ & & \\
actual body weight & $0.143 \pm 0.057$ & $0.039 \pm 0.017^{\mathrm{a}}$ \\
Inhibition rate & - & 70.85 \\
\hline
\end{tabular}

${ }^{a}$ The inhibition rate was calculated as described in Materials and methods. ${ }^{\text {b}} \mathrm{P}<0.01$.

Table II. Serum concentrations of cytokines.

\begin{tabular}{lcc}
\hline & \multicolumn{2}{c}{ Groups $(\mathrm{pg} / \mathrm{ml})$} \\
\cline { 2 - 3 } Cytokines & Control & Study \\
\hline IL-2 & $216.576 \pm 66.004$ & $912.878 \pm 72.036^{\mathrm{a}}$ \\
IL-4 & $30.257 \pm 8.327$ & $80.620 \pm 16.163^{\mathrm{a}}$ \\
IL-6 & $34.515 \pm 2.999$ & $82.946 \pm 13.628^{\mathrm{a}}$ \\
IL-8 & $57.285 \pm 8.785$ & $166.316 \pm 12.630^{\mathrm{a}}$ \\
IL-10 & $404.768 \pm 35.001$ & $673.272 \pm 40.305^{\mathrm{a}}$ \\
IL-12 & $0.995 \pm 0.198$ & $5.284 \pm 0.660^{\mathrm{a}}$ \\
IFN- $\gamma$ & $425.537 \pm 52.853$ & $503.201 \pm 43.922^{\mathrm{b}}$ \\
TNF- $\alpha$ & $217.007 \pm 33.128$ & $573.126 \pm 38.860^{\mathrm{a}}$ \\
TNF- $\beta$ & $66.628 \pm 5.625$ & $130.744 \pm 6.332^{\mathrm{a}}$ \\
G-CSF & $275.950 \pm 59.676$ & $758.219 \pm 69.910^{\mathrm{a}}$
\end{tabular}

${ }^{\mathrm{a}} \mathrm{P}<0.01 ;{ }^{\mathrm{b}} \mathrm{P}<0.05$. Data are presented as the mean $\pm \mathrm{SD}(\mathrm{n}=10)$. IL, interleukin; IFN, interferon; TNF, tumor necrosis factor; G-CSF, granulocyte-colony stimulating factor.

RNA isolation, library construction and miRNA sequencing. Total RNA was extracted from the dissected tumor tissues using the miRVana RNA isolation kit, while miRNA-enriched RNA fractions were isolated using the mirVana ${ }^{\mathrm{TM}}$ miRNA isolation kit, according to the manufacturer's instructions. RNA integrity was confirmed using Agilent 2100 Bioanalyzer small-RNA chips. Equal amounts of miRNA-enriched RNA fractions from five samples within the same group were combined and sent to the Genergy Biotech Shanghai Co., Ltd. (Shanghai, China) for small RNA library preparation. The libraries were then sequenced using the Solexa's Sequencing-By-Synthesis method, a massively parallel sequencing technology. The 36-bp reads on the Illumina Genome Analyzer (Illumina, Inc., Hayward, CA, USA) were used for sequencing.

Statistical analysis. miRNA expression levels were tested for statistically significant differences in the study and control group libraries using the Limma package (21). The Benjamini-Hochberg method (BH-FDR) (22) was used to 
Table III. Gene functional clusters enriched by DAVID annotation.

\begin{tabular}{lcc}
\hline Cluster term & Gene count, $\mathrm{n}^{\mathrm{a}}$ & P-value \\
\hline 1. Regulation of macromolecule biosynthetic process & 58 & $4.19 \mathrm{E}-25$ \\
2. Regulation of apoptosis & 31 & $2.76 \mathrm{E}-15$ \\
3. Regulation of transcription & 29 & $5.24 \mathrm{E}-9$ \\
4. Cell migration & 22 & $3.60 \mathrm{E}-8$ \\
5. Stem cell maintenance & 7 & $4.63 \mathrm{E}-6$ \\
6. Regulation of protein kinase activity & 16 & $9.17 \mathrm{E}-6$ \\
7. Regulation of caspase activity & 8 & $7.83 \mathrm{E}-5$ \\
8. Regulation of cell division & 6 & $8.81 \mathrm{E}-4$ \\
9. Glucose metabolic process & 12 & $1.82 \mathrm{E}-4$ \\
10. Cyclin-dependent protein kinase inhibitor activity & 4 & $1.75 \mathrm{E}-4$ \\
11. Transforming growth factor- $\beta$-related & 4 & 0.02 \\
12. Peptide hormone secretion & 4 & 0.03 \\
13. DNA fragmentation involved in apoptosis & 3 & 0.03 \\
14. Cellular ion homeostasis & 11 & 0.05 \\
15. Mitochondrial outer membrane & 5 & 0.05 \\
\hline
\end{tabular}

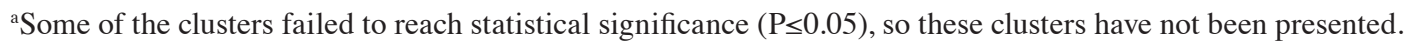

control the false discovery rate. Statistical significance was determined using the square root transformed templates per million (TPM) expression values of the 5-prime and 3-prime miRNA transcripts. Differences in expression with BH-FDR $\mathrm{P} \leq 0.05$ and a minimal 1.5 -fold change were considered to indicate a statistically significant difference.

Target selection and functional clustering. Target mRNAs for differentially expressed miRNAs were identified using miRWalk (http://www.rna.uni-heidelberg.de/apps/zmf/ mirwalk/index_html; last updated on March 15, 2011) in the validated targets module. Curated targets were subjected to functional enrichment to identify significantly over-represented biological functions using the DAVID functional clustering software (http://david.abcc.ncifcrf.gov). Functional clusters with statistical P-values $\leq 0.05$ were reported.

\section{Results}

Inhibitory effect of $\beta$-1,3-1,6-glucan on transplanted tumors. In the tumor intervention study, significant growth inhibition was observed in mice treated with $\beta-1,3-1,6$-glucan vs. the control mice, as demonstrated by slower tumor growth rates (Fig. 1) and reduced sizes of dissected tumors (Table I).

Changes in cytokine production. As shown in Table II, mice administered $\beta-1,3-1,6$-glucan demonstrated a significant increase in the levels of the tested cytokines, including IL-2, IL-4, IL-6, IL-8, IL-10, IL-12, IFN- $\gamma$, TNF- $\alpha$, TNF- $\beta$ and G-CSF. However, the elevation of serum IL-2 and IL-12 levels was more significant.

Changes in miRNA expression. Two datasets were obtained from two biological replicates in the study or control groups. Differentially expressed miRNAs with a minimum of 1.5-fold

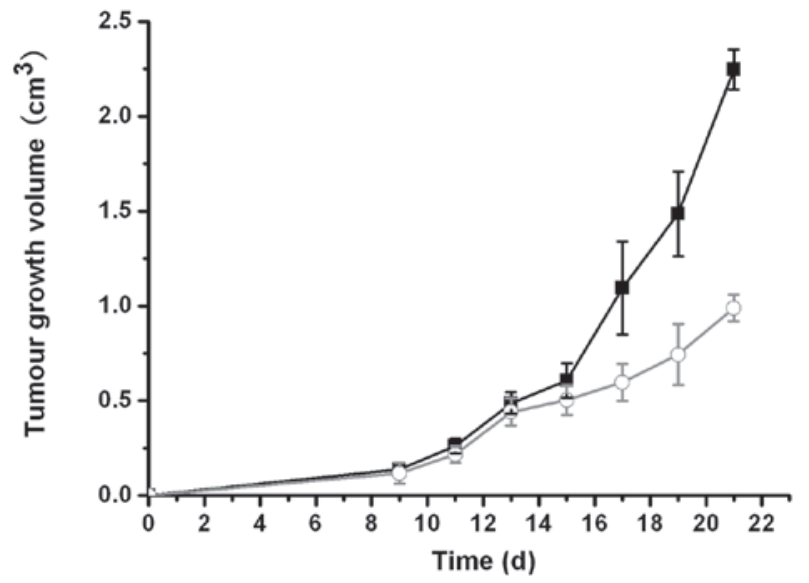

Figure 1. The growth curve of transplanted tumors is shown. The xenografted tumor sizes were measured daily in vivo using an external caliber. The data are expressed as the mean \pm standard deviation (SD). The open circles indicate tumor volumes in mice treated with $\beta$-1,3-1,6-glucan. The black squares indicate tumor volumes in control mice.

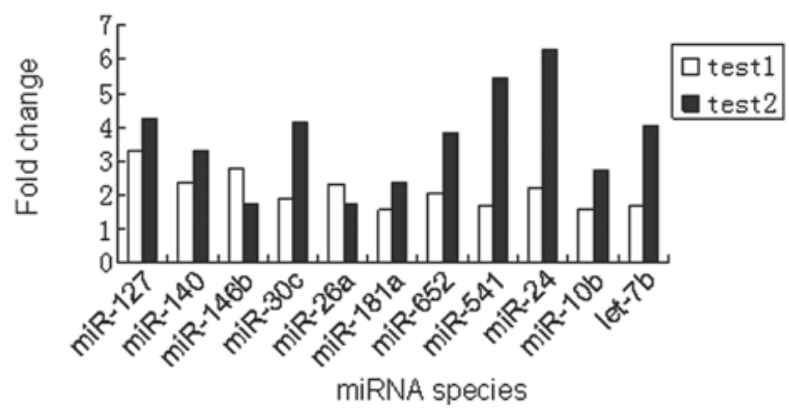

Figure 2. Differentially expressed miRNAs identified and quantified by massively parallel (Solexa) DNA sequencing are shown. The read counts of complemetary DNAs (cDNAs) derived from miRNAs in the libraries were calculated and then normalized to TPM. Differential levels of expression in the study and control groups are shown as ratios of TPM. Two individual experiments are reported. 
change were reported. Twelve miRNAs were reproducibly found to be upregulated in two biological replicates (Fig. 2).

Identification of target genes and functional enrichment. A total of 308 validated target genes were identified using the miRWalk database. These genes were classified using the DAVID functional annotation clustering program with the high-stringency enrichment algorithm. As a result, we retrieved 15 annotation clusters with $\mathrm{P} \leq 0.05$. These functional gene clusters are summarized in Table III. Notably, induction of apoptosis may contribute to the decrease in tumor growth and size.

\section{Discussion}

The antitumor effect of $\beta-1,3-1,6$-glucan has been documented in previous studies. The antitumor effects have been directly attributed to the activation of leukocytes. However, the incurred molecular and biochemical changes in tumor cells have not been fully elucidated. In this study, the antitumor efficacy of $\beta-1,3-1,6$-glucan derived from black yeast was assessed in subcutaneously-injected mice with mouse S180 sarcoma cells. Intragastric administration of $\beta-1,3-1,6$-glucan significantly decreased the growth rate of transplanted tumor cells and reduced tumor size during the 21-day treatment period. Mice administered $\beta$-1,3-1,6-glucan also demonstrated significantly higher levels of cytokines, such as IL-2, IL-4, IL-6, IL-8, IL-10, IL-12, IFN- $\gamma$, TNF- $\alpha$, TNF- $\beta$ and G-CSF, compared to the control mice. These findings demonstrate that $\beta-1,3-1,6$-glucan clearly triggers cytokine release in sarcoma xenograft-bearing mice. These findings are consistent with the data obtained from a previous study on a transplanted breast cancer model (23).

In addition, mice administered $\beta-1,3-1,6$-glucan also showed significant changes in the expression of several miRNAs in transplanted tumors. Previously, IL-6 was found to trigger the expression of miR-21 in myeloma cells (24). IL-6 was also shown to modulate the miR-17/92 cluster in human pulmonary artery endothelial (HPAE) cells through the STAT3 transcription factor (25). However, none of these miRNA species were found to be differentially expressed in the present study. Functional annotation clustering demonstrated that the target genes of these differentially expressed miRNAs are involved in the regulation of apoptosis and cell division. These findings indicate that $\beta-1,3-1,6$-glucan may exert its tumor-inhibition effects through the activation of the immune system and the modulation of miRNA expression in tumor cells.

Despite the limited number of studies available on $\beta$-glucans purified from certain plants having direct cytotoxic effects on cancer cells (16), the majority of the studies have shown no direct cytotoxic effects of fungal $\beta$-glucans on common cancer cell lines including carcinoma, sarcoma, blastoma $(1,26)$ or lymphoma (27). In the present study, we investigated the direct effects of $\beta-1,3-1,6$-glucan on the S180 sarcoma cell line in vitro and found no phenotypic changes in cell growth and apoptosis (data not shown). Thus, $\beta-1,3-1,6$-glucan inhibits the growth of transplanted tumors by direct activation of immunity and that alteration of miRNA expression is potentially induced by changes of cytokines indirectly. Previous studies have proven that the $\beta$-glucans exhibit their antitumor activity through stimulation of immune responses (26). The findings of this study suggest that $\beta-1,3-1,6$-glucan, extracted from black yeast, inhibits transplanted sarcoma through activation of the immune system as well as the modulation of miRNA expression in tumor cells. To the best of our knowledge, this is the first study investigating the effects of $\beta-1,3-1,6$-glucan ingestion on miRNA expression in transplanted tumor cells. The precise mechanisms of $\beta$-1,3-1,6-glucan-induced changes of miRNA expression remain to be fully elucidated.

\section{References}

1. Chan GC, Chan WK and Sze DM: The effects of beta-glucan on human immune and cancer cells. J Hematol Oncol 2: 25, 2009.

2. Tsoni SV and Brown GD: beta-Glucans and dectin-1. Ann N Y Acad Sci 1143: 45-60, 2008.

3. Schmid F, Stone BA, McDougall BM, Bacic A, Martin KL, Brownlee RT, Chai E and Seviour RJ: Structure of epiglucan, a highly side-chain/branched $(1 \rightarrow 3 ; 1 \rightarrow 6)-\beta$-glucan from the micro fungus Epicoccum nigrum Ehrenb. ex Schlecht. Carbohydr Res 331: 163-171, 2001.

4. Chen J and Seviour R: Medicinal importance of fungal beta-( $(1 \rightarrow 3),(1 \rightarrow 6)$-glucans. Mycol Res 111: 635-652, 2007.

5. Rop O, Mlcek J and Jurikova T: Beta-glucans in higher fungi and their health effects. Nutr Rev 67: 624-631, 2009.

6. Ariizumi K, Shen GL, Shikano S, Xu S, Ritter R III, Kumamoto T, Edelbaum D, Morita A, Bergstresser PR and Takashima A: Identification of a novel, dendritic cell-associated molecule, dectin-1, by subtractive cDNA cloning. J Biol Chem 275: 20157-20167, 2000 .

7. Brown GD and Gordon S: Immune recognition. A new receptor for beta-glucans. Nature 413: 36-37, 2001.

8. Ross GD: Regulation of the adhesion versus cytotoxic functions of the Mac-1/CR3/alphaMbeta2-integrin glycoprotein. Crit Rev Immunol 20: 197-222, 2000.

9. Lebron F, Vassallo R, Puri V and Limper AH: Pneumocystis carinii cell wall beta-glucans initiate macrophage inflammatory responses through NF-kappaB activation. J Biol Chem 278: 25001-25008, 2003.

10. Acton SL, Scherer PE, Lodish HF and Krieger M: Expression cloning of SR-BI, a CD36-related class B scavenger receptor. J Biol Chem 269: 21003-21009, 1994.

11. Rice PJ, Kelley JL, Kogan G, Ensley HE, Kalbfleisch JH, Browder IW and Williams DL: Human monocyte scavenger receptors are pattern recognition receptors for $(1 \rightarrow 3)$-beta-D-glucans. J Leukoc Biol 72: 140-146, 2002.

12. Zimmerman JW, Lindermuth J,Fish PA, Palace GP, Stevenson TT and DeMong DE: A novel carbohydrate-glycosphingolipid interaction between a beta-(1-3)-glucan immunomodulator, PGG-glucan, and lactosylceramide of human leukocytes. J Biol Chem 273: 22014-22020, 1998.

13. Vetvicka V and Yvin JC: Effects of marine beta-1,3 glucan on immune reactions. Int Immunopharmacol 4: 721-730, 2004.

14. Mizuno M, Minato K, Ito $\mathrm{H}$, Kawade M, Terai $\mathrm{H}$ and Tsuchida $\mathrm{H}$ : Anti-tumor polysaccharide from the mycelium of liquid-cultured Agaricus blazei mill. Biochem Mol Biol Int 47: 707-714, 1999.

15. Cheung NK and Modak S: Oral $(1 \rightarrow 3),(1 \rightarrow 4)$-beta-D-glucan synergizes with antiganglioside GD2 monoclonal antibody $3 \mathrm{~F} 8$ in the therapy of neuroblastoma. Clin Cancer Res 8: 1217-1223, 2002.

16. Zhang M, Chiu LC, Cheung PC and Ooi VE: Growth-inhibitory effects of a $\beta$-glucan from the mycelium of Poria cocos on human breast carcinoma MCF-7 cells: Cell-cycle arrest and apoptosis induction. Oncol Rep 15: 637-643, 2006.

17. Kimura Y, Sumiyoshi M, Suzuki T and Sakanaka M: Antitumor and antimetastatic activity of a novel water-soluble low molecular weight beta-1, 3-D-glucan (branch beta-1,6) isolated from Aureobasidium pullulans 1A1 strain black yeast. Anticancer Res 26: 4131-4141, 2006.

18. Ikewaki N, Fujii N, Onaka T, Ikewaki S and Inoko H: Immunological actions of Sophy beta-glucan (beta-1,3-1,6 glucan), currently available commercially as a health food supplement. Microbiol Immunol 51: 861-873, 2007.

19. Euhus DM, Hudd C, LaRegina MC and Johnson FE: Tumor measurement in the nude mouse. J Surg Oncol 31: 229-234, 1986. 
20. Tomayko MM and Reynolds CP: Determination of subcutaneous tumor size in athymic (nude) mice. Cancer Chemother Pharmacol 24: 148-154, 1989.

21. Smyth GK: Linear models and empirical bayes methods for assessing differential expression in microarray experiments. Stat Appl Genet Mol Biol 3: Article3, 2004.

22. Reiner A, Yekutieli D and Benjamini Y: Identifying differentially expressed genes using false discovery rate controlling procedures. Bioinformatics 19: 368-375, 2003.

23. Vetvicka V, Vashishta A, Saraswat-Ohri S and Vetvickova J: Immunological effects of yeast- and mushroom-derived beta-glucans. J Med Food 11: 615-622, 2008.

24. Löffler D, Brocke-Heidrich K, Pfeifer G, Stocsits C, Hackermüller J, Kretzschmar AK, Burger R, Gramatzki M, Blumert C, Bauer K, et al: Interleukin-6 dependent survival of multiple myeloma cells involves the Stat3-mediated induction of microRNA-21 through a highly conserved enhancer. Blood 110 $1330-1333,2007$
25. Brock M, Trenkmann M, Gay RE, Michel BA, Gay S, Fischler M, Ulrich S, Speich R and Huber LC: Interleukin-6 modulates the expression of the bone morphogenic protein receptor type II through a novel STAT3-microRNA cluster 17/92 pathway. Circ Res 104: 1184-1191, 2009.

26. Gomaa K, Kraus J, Rosskopf F, Röper H and Franz G: Antitumour and immunological activity of a beta $1 \rightarrow 3 / 1 \rightarrow 6$ glucan from Glomerella cingulata. J Cancer Res Clin Oncol 118: 136-140, 1992.

27. Harnack U, Kellermann U and Pecher G: Yeast-derived beta-(1-3),(1-6)-D-glucan induces up-regulation of CD86 on dectin-1-positive human B-lymphoma cell lines. Anticancer Res 31: 4195-4199, 2011. 